\title{
OTIMIZAÇÃO DAS CONDIÇÕES DE PRÉ-REDUÇÃO DO As(V) EM EXTRATOS DO MÉTODO BCR PARA QUANTIFICAÇÃO DE As POR HG-AAS ${ }^{(1)}$
}

\author{
Eduardo Vinícius Vieira Varejão ${ }^{(2)}$, Carlos Roberto Bellato ${ }^{(3)}$, Jaime \\ Wilson Vargas de Mello ${ }^{(4)}$ \& Maurício Paulo Ferreira Fontes ${ }^{(5)}$
}

\begin{abstract}
RESUMO
A determinação de As por espectrometria de absorção atômica com geração de hidretos (HG-AAS) constitui um método simples, sensível, preciso e de baixo custo. Entretanto, essa técnica requer a pré-redução das espécies de $\mathrm{As}(\mathrm{V})$, o que se obtém através do uso de agentes redutores como o KI. Em extratos contendo agentes oxidantes, a pré-redução do As é comprometida, como acontece em extratos obtidos pela aplicação do método BCR (acrônimo francês para Community Bureau of Reference) para a extração sequencial de As em sedimentos. O objetivo deste trabalho foi avaliar as condições de redução do As(V) a As(III), de modo a permitir o uso da HG-AAS para a quantificação do arsênio em extratos obtidos a partir do método BCR. Foram avaliadas condições reacionais utilizando KI, L-cisteína e ácido ascórbico. Para cada uma das etapas de extração do método BCR, diferentes condições de pré-redução possibilitaram a detecção quantitativa do As presente. O uso do método BCR para a extração de arsênio em amostras de sedimentos contaminadas e a aplicação das condições de pré-redução do As(V) selecionadas, seguida pela detecção por HG-AAS, forneceram percentagens de recuperação entre 91 e $99 \%$.
\end{abstract}

Termos de indexação: arsênio, extração sequencial, Espectrometria de Absorção Atômica com Geração de Hidretos, sedimentos.

\footnotetext{
(1) Parte da Dissertação de Mestrado do primeiro autor apresentada ao Programa de Pós-Graduação em Agroquímica, Universidade Federal de Viçosa - UFV. Recebido para publicação em outubro de 2008 e aprovado em maio de 2009.

(2) Doutorando em Agroquímica, Universidade Federal de Viçosa - UFV. Av. PH Holfs s/n, CEP 36570-000 Viçosa (MG). E-mail: eduardo.varejao@ufv.br

(3) Professor Associado do Departamento de Química, UFV. E-mail: bellato@ufv.br

(4) Professor Associado do Departamento de Solos, UFV. E-mail: jwvmello@ufv.br

(5) Professor Titular do Departamento de Solos, UFV. E-mail: mpfontes@ufv.br
} 


\title{
SUMMARY: OPTIMIZATION OF PRE-REDUCTION CONDITIONS OF AS(V) IN BCR EXTRACTS TO QUANTIFY ARSENIC BY HG-AAS
}

\begin{abstract}
The determination of As by hydride generation atomic absorption spectroscopy (HG$A A S)$ is a simple, sensitive, precise and low-cost method. However, this technique requires the pre-reduction of the existing As(V) species, which is obtained by the use of reducing agents such as KI. In extracts containing oxidizing agents, the pre-reduction of As is impaired, as it occurs in extracts obtained by the BCR (French acronym for Community Bureau of Reference) method for the sequential extraction of As in sediments. The objective of this study was to evaluate the conditions for the reduction of $A s(V)$ to As(III) in a way that allows the use of HG-AAS for the quantification of arsenic in extracts obtained using the BCR method. Reaction conditions were evaluated using KI, L-Cysteine and ascorbic acid. For each of the BCR extraction steps, different conditions of pre-reduction enabled the quantitative detection of As. The use of the $B C R$ method for the extraction of arsenic from contaminated sediment samples and the application of pre-reduction conditions of the As $(V)$ followed by $H G$-AAS detection resulted in relative recoveries between 91 and $99 \%$.
\end{abstract}

Index terms: arsenic, sequential extraction, hydride generation atomic absorption spectroscopy, sediments.

\section{INTRODUÇÃO}

A Geração de Hidretos (HG), acoplada a diferentes técnicas de espectrometria atômica, tem sido amplamente utilizada para análises de arsênio em diferentes matrizes, incluindo solos e sedimentos. Entre os diferentes métodos espectrométricos de quantificação, a absorção atômica (AAS) tem sido a mais utilizada, constituindo uma técnica analítica simples, sensível, precisa e rápida. O baixo custo da técnica permite que os espectrômetros de absorção atômica encontrem-se disponíveis na maioria dos laboratórios. Essas vantagens fazem com que a HGAAS permaneça como a melhor escolha para a quantificação de arsênio para vários tipos de amostras (Shraim et al., 1999; Takase et al., 2002; Kumar \& Riyazuddin, 2005).

A técnica de HG-AAS baseia-se na redução dos compostos de As por boroidreto de sódio $\left(\mathrm{NaBH}_{4}\right)$ em meio ácido. $\mathrm{O} \mathrm{NaBH}_{4}$ volatiliza as espécies inorgânicas de arsênio por meio da formação de $\mathrm{AsH}_{3}$ (arsina) e os compostos metilados de arsênio $\mathrm{Me}_{\mathrm{n}} \mathrm{AsO}(\mathrm{OH})_{3-n}(\mathrm{n}=1$ a 3) pela formação dos hidretos de metilarsênio(III), dimetilarsênio(III) e trimetilarsênio(III) (Kumaresan \& Riyazuddin, 2001). A formação de $\mathrm{AsH}_{3}$ volátil permite a separação prévia do analito a partir da amostra e facilita o transporte até o sistema de atomização. O sistema consiste de um tubo de quartzo em forma de $\mathrm{T}$ alinhado no caminho óptico e com o braço central servindo de passagem ao hidreto e ao gás carreador para o interior da parte aquecida do tubo. A volatilização e a separação prévia do arsênio diminuem as interferências e aumentam a sensibilidade e a seletividade do método (Takase et al., 2002).

Há, entretanto, um forte efeito do estado de oxidação do arsênio na formação do hidreto volátil. A velocidade da reação do As(III) com o $\mathrm{NaBH}_{4}$ é muito maior que a do $\mathrm{As}(\mathrm{V})$, e a cinética de reação mais lenta do $\mathrm{As}(\mathrm{V})$ com o $\mathrm{NaBH}_{4}$ leva a picos largos e pequenos, de modo que a intensidade do pico de absorção para o hidreto de $\mathrm{As}(\mathrm{V})\left(\mathrm{AsH}_{5}\right)$ é significativamente menor do que para $\mathrm{o} \mathrm{AsH}_{3}$, o que leva à subestimativa das quantidades de As presentes (Feng et al., 1998; Boutakhrit et. al., 2005). Dessa forma, faz-se necessário proceder à completa pré-redução do $\mathrm{As}(\mathrm{V})$ a As(III) antes do contato com a solução de $\mathrm{NaBH}_{4}$, o que tem sido obtido pelo uso de iodeto de potássio (KI) como agente redutor (Burguera \& Burguera, 1993; Dedina, \& Tsalev, 1995; Creed et al., 1996; Risnes \& Lund, 1996; Takase et al., 2002).

A técnica torna-se limitada quando a extração de arsênio a partir de solos ou sedimentos é obtida pelo uso de agentes extratores oxidantes. Uma vez presentes no extrato final, reagentes oxidantes reagem com o iodeto utilizado para a pré-redução do $\mathrm{As}(\mathrm{V})$. Com o consumo do iodeto pelo agente extrator, há o comprometimento da redução quantitativa do $\mathrm{As}(\mathrm{V})$ a As(III), além da formação de quantidades excessivas de iodo, o qual interfere na formação e na liberação do hidreto (Barra et al., 2000; Takase et al., 2002).

Alguns agentes oxidantes são comumente utilizados como extratores em métodos de extração em etapa única ou em métodos de extração sequencial. Os métodos de extração sequencial são desenvolvidos para permitir a extração "seletiva" de elementos-traço a partir de frações dos sedimentos operacionalmente definidas, fornecendo informações mais detalhadas acerca das quantidades dos elementos-traço associadas aos diferentes compartimentos do sedimento (Tessier et al., 1979; Davidson et al., 1998; Pueyo et al., 2003; Zemberyová et al., 2006). Como as frações extraídas em um procedimento sequencial são 
operacionalmente definidas, as diferentes condições químicas utilizadas em cada etapa dos diferentes métodos semelhantes dificultam a comparação de resultados obtidos por métodos diferentes, a avaliação da eficiência de extração e a validação dos diversos métodos (Hlavay et al., 2004; Marguí et al., 2004). Para padronizar os procedimentos e permitir a comparação e o controle de qualidade, a instituição europeia Community Bureau of Reference (Bureau Communautaire de Référence, BCR) desenvolveu um método para a determinação de metais-traço em solos e sedimentos. Um procedimento padronizado de extração em três etapas (BCR EUR $1473 \mathrm{EN}$ ) foi proposto para a análise de elementos-traço em sedimentos (Ure et al., 1993; Rauret, 1999). O método vem sendo amplamente utilizado em diversas pesquisas sobre a distribuição de metais-traço e arsênio em sedimentos (Dhoun \& Evans, 1998; Sahuquillo et al., 1999; Weisz et al., 2000; Tokalioglu et al., 2000; Marguí et al., 2004; Basílio et al., 2005; Zemberyová et al., 2006; Pertsemli \& Voutusa, 2007; Pereira et al., 2007).

$\mathrm{O}$ uso de agentes oxidantes como extratores nas etapas 3 e 4 da extração pelo método BCR interfere na redução do $\mathrm{As}(\mathrm{V})$ e na detecção quantitativa do arsênio presente nos extratos, devido ao consumo do iodeto e à consequente produção de iodo $\left(\mathrm{I}_{3}^{-}\right)$em quantidade elevada. Dessa forma, este trabalho teve como objetivo avaliar diferentes condições de pré-redução do As(V) nos extratos obtidos pela aplicação do método BCR na extração sequencial de arsênio em sedimentos de forma a se obter condições que permitam a quantificação do As por HG-AAS.

\section{MATERIAL E MÉTODOS}

\section{Equipamentos, reagentes e soluções}

As massas das amostras e de reagentes foram medidas em balança METTLER TOLEDO, modelo AB204-S, com precisão de 0,0001 g. Para a extração sequencial, foram utilizados agitador mecânico, marca CERTOMAT MO, banho Dubnoff Microprocessado QUMIS Q-226M, centrífuga FANEM, modelo 315, e forno de microondas MILESTONE modelo Ethosplus. A determinação do arsênio foi realizada por meio de espectrômetro de absorção atômica (VARIAN, modelo spectrAA 200) com gerador de hidretos (VARIAN, modelo VGA 77).

Todos os reagentes utilizados neste estudo foram de grau analítico, marcas VETEC e MERCK. Para o preparo das soluções e lavagem de frascos e vidraria, utilizou-se água deionizada previamente destilada. Todos os frascos utilizados foram imersos em $\mathrm{HNO}_{3}$ $10 \%(\mathrm{v} / \mathrm{v})$ por $48 \mathrm{~h}$ e, posteriormente, enxaguados exaustivamente.

\section{Procedimento de extração sequencial}

O protocolo BCR compreende quatro etapas de extração (Quadro 1) (Ure et al., 1993; Rauret et al., 1999). Embora o procedimento preconize o uso de $1.0000 \mathrm{~g}$ de sedimento, diversos autores têm aplicado o método utilizando diferentes valores de massa. Os resultados obtidos são perfeitamente satisfatórios (Marguí et al., 2004). Deve-se, entretanto, observar a proporcionalidade entre a massa de sedimento utilizada e os volumes dos extratores empregados, já que modificações dessas razões implicam alterações dos resultados obtidos (Davidson et al., 1998).

Neste trabalho, exatamente $0,8000 \mathrm{~g}$ de sedimento foi submetido à extração. Todas as análises foram feitas em triplicata.

A classificação operacional das frações extraídas por cada etapa e os respectivos minerais-alvo, aos quais estão ligados os elementos extraídos em cada etapa, encontram-se dispostos no quadro 2 (Davidson et al., 1998; Gismera et al., 2004):

\section{Amostras de sedimentos}

As amostras de sedimentos utilizadas foram coletadas em quatro sítios de amostragem nos ribeirões

\section{Quadro 1. Protocolo BCR de extração sequencial}

\begin{tabular}{|c|c|c|c|c|c|}
\hline Etapa & Extrator & Volume & Temperatura & Tempo & Agitação \\
\hline & & $\mathrm{mL}^{(1)}$ & ${ }^{\circ} \mathrm{C}$ & $\mathrm{h}$ & \\
\hline 1 & $\mathrm{CH}_{3} \mathrm{COOH} 0,11 \mathrm{~mol} \mathrm{~L}^{-1}$ & 32 & $22 \pm 5$ & 16 & $40 \mathrm{rpm}$ \\
\hline 2 & $\mathrm{NH}_{2} \mathrm{OH} . \mathrm{HCl} 0,5 \mathrm{~mol} \mathrm{~L}^{-1}(\mathrm{pH} 1,5)$ & 32 & $22 \pm 5$ & 16 & $40 \mathrm{rpm}$ \\
\hline \multirow[t]{4}{*}{3} & $\mathrm{H}_{2} \mathrm{O}_{2} 8,8 \mathrm{~mol} \mathrm{~L}^{-1}(\mathrm{pH} 2)^{(2)}$ & 8 & $22 \pm 5$ & 1 & ocasional \\
\hline & & & $80 \pm 5$ & 1 & ocasional \\
\hline & $\mathrm{H}_{2} \mathrm{O}_{2} 8,8 \mathrm{~mol} \mathrm{~L}^{-1}(\mathrm{pH} 2)^{(2)}$ & 8 & $80 \pm 5$ & 1 & ocasional \\
\hline & $\mathrm{NH}_{4} \mathrm{OAc} 1 \mathrm{~mol} \mathrm{~L}^{-1}(\mathrm{pH} 2)^{(2)}$ & 40 & $22 \pm 5$ & 16 & $40 \mathrm{rpm}$ \\
\hline 4 & Água regia $\left(\mathrm{HCl} / \mathrm{HNO}_{3} 3: 1\right)$ & 10 & Digestão á & em forno $d$ & icroondas \\
\hline
\end{tabular}

\footnotetext{
${ }^{(1)}$ Em relação a uma massa de $0,8 \mathrm{~g}$ de sedimento seco. ${ }^{(2)}$ Valores de $\mathrm{pH}$ ajustados com ácido nítrico concentrado.
} 
Tripuí e do Carmo, localizados no Quadrilátero Ferrífero, entre os municípios de Ouro Preto e Mariana, Minas Gerais. O Quadrilátero Ferrífero é conhecido como um dos maiores depósitos minerais do planeta. Além de grandes depósitos de ferro, diversas mineralizações auríferas sulfetadas estão presentes, com as quais o As tem importante correlação. Os principais minerais sulfetados presentes são a arsenopirita e a pirita enriquecida em As. Em alguns depósitos, a relação As/Au varia de 300 a 3.000. A região é reconhecida como a mais antiga e importante província aurífera do Brasil, desde meados do século XVII. As históricas atividades de mineração desenvolvidas na região têm sido responsáveis pela liberação de grandes quantidades de As para ambientes aquáticos e terrestres. Elevados teores de As têm sido registrados em água, solos e sedimentos desta região, relacionados tanto à litologia local quanto ao descarte de rejeitos proveniente das atividades de mineração (Matschullat et al., 2000; Deschamps et al., 2002; Borba et al., 2003; Mello et al., 2006; Gonçalves et al., 2007).

Os procedimentos de extração, pré-redução do $\mathrm{As}(\mathrm{V})$ e quantificação por HG-AAS foram aplicados às amostras de sedimentos coletadas, bem como o material de referência SRM 2704 Buffalo River Sediment (NIST - National Institute of Standard and Technology, USA) com teor total de As certificado.

\section{Estudo da Redução do As(V) a As(III) nos extratos}

A redução do $\mathrm{As}(\mathrm{V})$ a $\mathrm{As}(\mathrm{III})$, antes da reação com o $\mathrm{NaBH}_{4}$ para a formação da arsina, foi avaliada sob três condições distintas: L-cisteína a 5,0\% $(\mathrm{m} / \mathrm{v}), \mathrm{KI}$ $1,0 \%(\mathrm{~m} / \mathrm{v})$ e KI $1,0 \%(\mathrm{~m} / \mathrm{v})$ associado ao ácido ascórbico nas concentrações de 1,0;2,0; 3,0; e 4,0 \% $(\mathrm{m} / \mathrm{v})$. O tempo de reação foi de $15 \mathrm{~min}$ para todas as condições de redução avaliadas e as reações se deram em meio de $\mathrm{HCl} 1 \mathrm{~mol} \mathrm{~L}^{-1}$. Os extratos foram transferidos para balões de $50,00 \mathrm{~mL}$ e o volume completado até a marca. Desse volume, $25,00 \mathrm{~mL}$ foram transferidos para outro balão de $50,00 \mathrm{~mL}$ foram adicionados volumes necessários de $\mathrm{HCl}$ concentrado $\left(1,19 \mathrm{~g} \mathrm{~mL}^{-1}, 35 \%(\mathrm{~m} / \mathrm{m})\right)$ para obter concentração final de $1 \mathrm{~mol} \mathrm{~L}^{-1} \mathrm{em} \mathrm{HCl}$, e volumes necessários de

Quadro 2. Frações operacionalmente definidas e fases-alvo de cada etapa do método BCR de extração sequencial

\begin{tabular}{cll}
\hline $\begin{array}{c}\text { Etapa de } \\
\text { extração }\end{array}$ & $\begin{array}{c}\text { Fração } \\
\text { extraída }\end{array}$ & \multicolumn{1}{c}{ Fase alvo } \\
\hline 1 & Ácido-solúvel & Solúvel + carbonatos \\
2 & Redutível & (Oxi) hidróxidos de Fe e Mn \\
3 & Oxidável & Sulfetos e matéria orgânica \\
Pseudototal & Residual & Minerais não silicatados \\
\hline
\end{tabular}

soluções de L-cisteína, KI e ácido ascórbico, de modo a conseguir as concentrações finais desejadas. Os volumes foram completados para $50,00 \mathrm{~mL}$ com água deionizada.

Após a seleção inicial das condições de pré-redução para cada uma das etapas da extração, foram preparadas soluções de As(V) nas concentrações de 1,0; 5,$0 ; 10,0 ; 20,0 ; 30,0$; e 50,0 $\mathrm{g} \mathrm{L} \mathrm{L}^{-1}$ contendo os extratores químicos utilizados em cada uma das etapas do método de extração sequencial separadamente, de modo a simular as condições químicas presentes nos extratos finais provenientes da aplicação do método BCR na extração de As de amostras de sedimentos. As quantidades de arsênio detectadas a partir das soluções de $\mathrm{As}(\mathrm{V})$ foram comparadas com as quantidades detectadas a partir de soluções-padrão de As(III) nas mesmas condições, a fim de se comprovar a eficiência das condições pré-redutoras selecionadas.

Quando do uso da L-cisteína, a formação dos hidretos se deu em meio de $\mathrm{HCl} 8 \mathrm{~mol} \mathrm{~L}^{-1}$, utilizando como agente redutor solução de $\mathrm{NaBH}_{4} 0,6 \%$ (m/v) estabilizada em $\mathrm{NaOH}$ 0,1 \% (m/v) (Shraim et al., 1999). Quando KI ou KI/ácido ascórbico foram utilizados, os hidretos foram gerados por reação com $\mathrm{NaBH}_{4} 0,4 \%(\mathrm{~m} / \mathrm{v})$ estabilizada em NaOH 0,5\% (m/v), em meio de HCl 6 mol L-1 (Ward \& Gray, 1994; Nielsen \& Hansen, 1997).

\section{Curvas analíticas e limites de detecção}

As curvas analíticas foram preparadas contendo os mesmos reagentes utilizados nas extrações e na pré-redução do As(V), nas mesmas concentrações, para cada uma das etapas. Os limites de detecção para o arsênio foram calculados para cada uma das etapas, com base no critério $3 \sigma$ (Long \& Winefordner, 1983; Corley, 2003):

\section{RESULTADOS E DISCUSSÃO}

\section{Avaliação da redução do $\mathrm{As}(\mathrm{V})$ nos extratos do método BCR}

Nos extratos provenientes das etapas 1 e 2 da extração sequencial, não foi observada diferença significativa entre as quantidades de As detectadas em função das diferentes condições redutoras para o As(V) (Figura 1).

Na primeira etapa, obteve-se a liberação do As associado a carbonatos através da dissolução desses minerais por ação do ácido acético, utilizado como extrator. $\mathrm{Na}$ etapa seguinte, o agente redutor cloridrato de hidroxilamina foi utilizado para a dissolução redutiva de (oxi)hidróxidos de Fe e Mn, liberando o As associado a estas frações. Como ambos os extratores não apresentam propriedades oxidativas, a presença destes reagentes nos extratos finais não interferiu na reação entre o iodeto ou a L-cisteína e o As(V). Quando da utilização do KI na ausência de ácido 

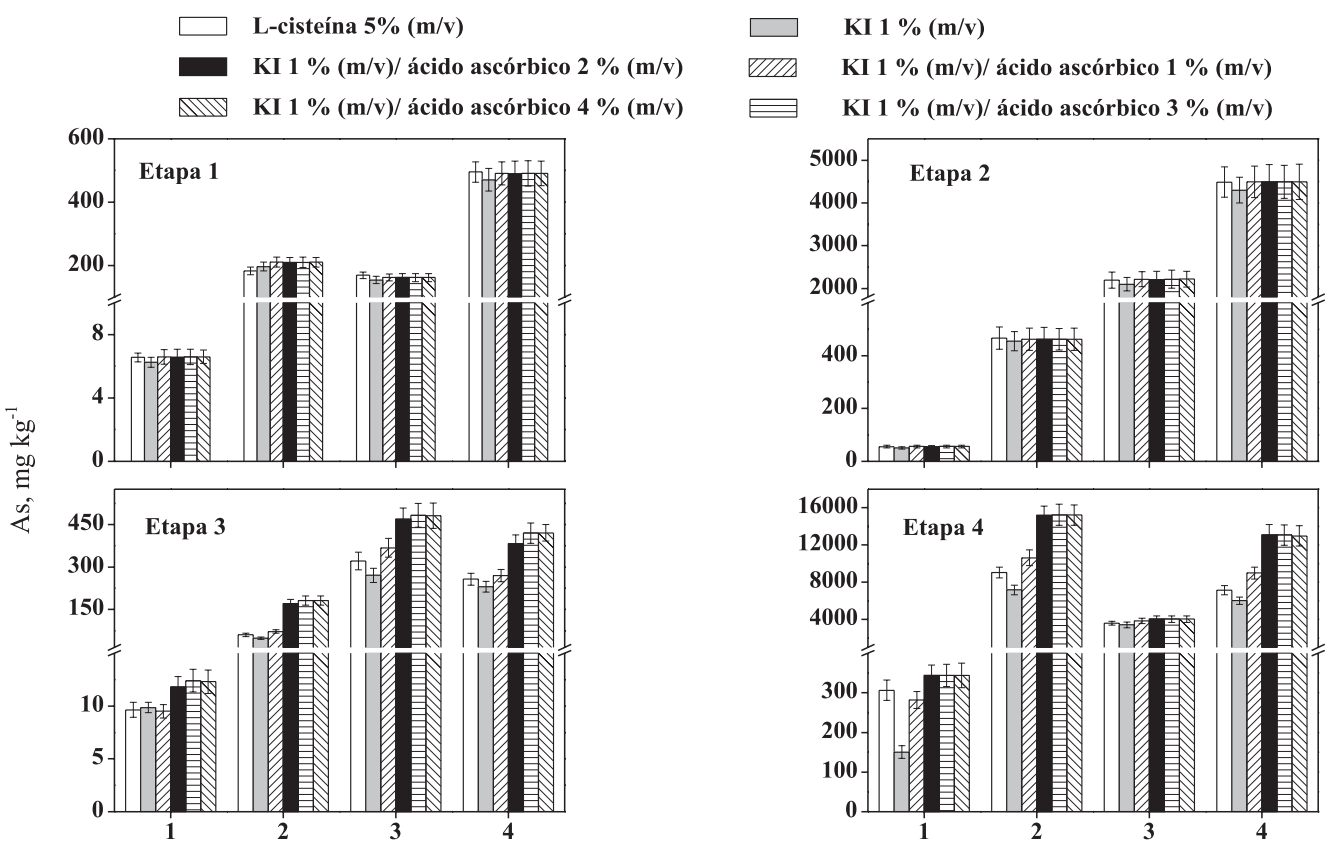

AMOSTRA DE SEDIMENTO

Figura 1. Teores de As nos extratos obtidos pela aplicação do método BCR de extração sequencial em quatro amostras de sedimentos, em função das condições de pré-redução do As(V) utilizadas.

ascórbico, apenas uma pequena quantidade de iodo foi formada na solução, o que se pôde presumir pelo desenvolvimento de coloração apenas levemente púrpura no meio reacional. Esta quantidade de iodo formado provém da própria reação entre o iodeto e o $\mathrm{As}(\mathrm{V})$, e não interferiu na reação entre As(III) formado e o $\mathrm{NaBH}_{4}$.

Como descrito na literatura, o uso do KI para a redução do $\mathrm{As}(\mathrm{V})$ na presença de agentes oxidantes fortes resulta na produção de grandes quantidades de iodo. Esta situação foi observada quando do uso do KI sem adição de ácido ascórbico, nos extratos das etapas 3 e 4 da extração sequencial, o que se deve à presença dos extratores oxidantes $\mathrm{H}_{2} \mathrm{O}_{2}$ e água régia, respectivamente, nas soluções finais. Nos extratos da terceira etapa, grandes quantidades de precipitado de iodo se formaram. Pela análise dos gráficos da figura 1, pode-se verificar que o uso do KI e da Lcisteína para reduzir o $\mathrm{As}(\mathrm{V})$ nesses extratos levou à detecção de quantidades menores de As, devido ao consumo destes agentes redutores pelos extratores oxidantes. A adição de ácido ascórbico foi capaz de evitar a oxidação do iodeto e a formação de grandes quantidades de iodo. Entretanto, o ácido ascórbico só foi plenamente eficiente a partir de uma concentração final de 3,0 \% (m/v). Concentrações menores de ácido ascórbico nas soluções finais da etapa 3 não foram suficientes para prevenir a oxidação do iodeto, e quantidades acima de $3,0 \%(\mathrm{~m} / \mathrm{v})$ não implicaram aumento da quantidade de As detectado por HG-AAS. Para a etapa 4, a adição de ácido ascórbico em concentração final de $2,0 \%(\mathrm{~m} / \mathrm{v})$ foi suficiente para prevenir a oxidação do iodeto. Em concentrações menores que $2,0 \%(\mathrm{~m} / \mathrm{v})$, houve ainda o desenvolvimento de coloração púrpura nas soluções e a detecção de quantidades inferiores de As.

Pelos resultados obtidos em princípio, a redução do As(V) nos extratos do BCR poderia ser então obtida de modo eficaz utilizando-se KI 1,0 \% (m/v) nos extratos das etapas 1 e 2, e KI 1,0 \% (m/v) associado a ácido ascórbico nas concentrações finais de 3,0 e 2,0 \% $(\mathrm{m} / \mathrm{v})$ nos extratos das etapas 3 e 4 , respectivamente. Embora a L-cisteína também tenha se mostrado eficaz para a redução do $\mathrm{As}(\mathrm{V})$ nos extratos das etapas 1 e 2, o KI foi selecionado como pré-redutor nestas etapas por motivo de simplificação da metodologia pela utilização do mesmo reagente em todas as etapas do método.

As condições de pré-redução selecionadas foram aplicadas a soluções de concentrações conhecidas de $\mathrm{As}(\mathrm{V})$ contendo os extratores químicos utilizados em cada uma das etapas do método de extração sequencial separadamente, de modo a simular as condições químicas presentes nos extratos finais provenientes da aplicação do método BCR na extração de As de amostras de sedimentos. Soluções-padrão de As(III) foram preparadas nas mesmas condições, e as quantidades de As detectadas a partir das soluções de $\mathrm{As}(\mathrm{V})$ foram plotadas contra as quantidades detectadas a partir das soluções de As(III) (Figura 2). Verifica-se boa correlação entre os resultados obtidos a partir da solução de As(V) e das soluções-padrão de As(III), mostrando que as condições de pré-redução utilizadas foram capazes de garantir a recuperação quantitativa do As $(V)$ nas soluções. 

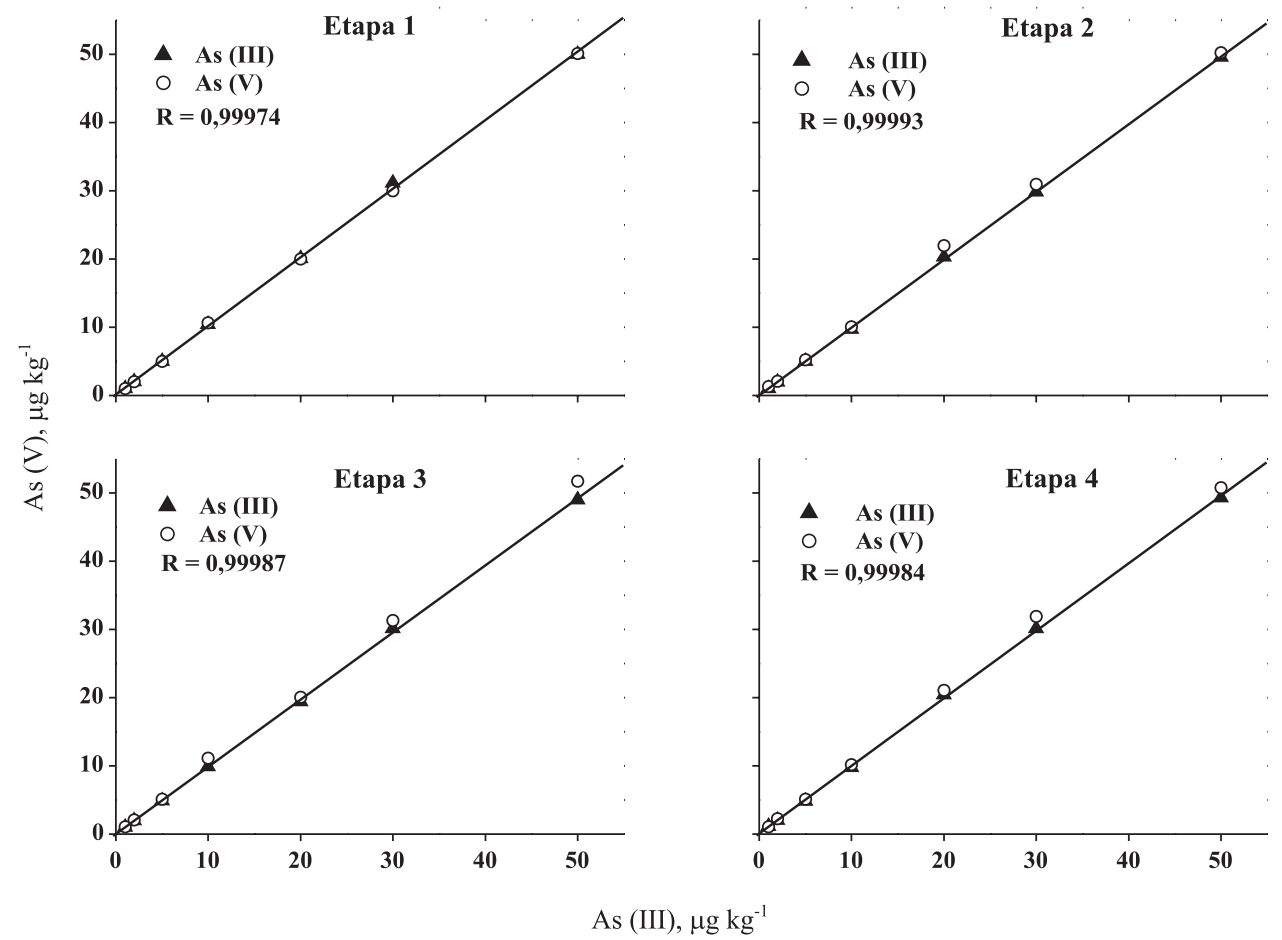

Figura 2. Correlação entre As recuperado a partir de soluções de As(V) tratadas com as condições préredutoras selecionadas para cada etapa do método de extração sequencial e soluções-padrão de As(III), nas mesmas condições.

Os limites de detecção para a análise de As nos extratos provenientes das etapas 1, 2, 3 e 4 foram $1,35,1,83,1,86$ e $1,42 \mu \mathrm{g} \mathrm{L}^{-1}$, respectivamente.

\section{Avaliação da recuperação do As a partir dos sedimentos}

O método BCR de extração sequencial consiste de um procedimento operacionalmente definido, no qual, em cada uma das etapas, os reagentes são utilizados com o objetivo de liberar os elementos-traço associados aos diferentes componentes geoquímicos do sedimento. As fases-alvo de cada uma das etapas do método BCR são: carbonatos e elementos trocáveis (etapa 1); (oxi)hidróxidos de Fe e Mn (etapa 2); matéria orgânica e sulfetos (etapa 3); e minerais não-silicatados remanescentes (etapa 4). Os elementos liberados nas etapas de 1 a 4 são denominados fração ácido-solúvel, redutível, oxidável e residual, respectivamente. A digestão de uma alíquota do sedimento integral pelo uso de água régia é considerada como digestão pseudototal, por não liberar a fração dos elementos de interesse presentes nas estruturas cristalinas de minerais silicatados. A digestão pseudototal do sedimento integral (não submetido às etapas da extração sequencial) permite o cálculo da porcentagem de recuperação do método, obtida pelo quociente da soma das quantidades do elemento extraídas nas 4 etapas e o teor pseudototal. A porcentagem de recuperação funciona como método interno de checagem da capacidade de recuperação dos elementostraço.
Além do cálculo da porcentagem de recuperação, a eficiência do método foi avaliada pela aplicação de todos os procedimentos (extração, pré-redução do $\mathrm{As}(\mathrm{V})$ e determinação por HG-AAS) em material de referência SRM 2704 NIST (Buffalo River Sediment), com valor total de As certificado.

As porcentagens de recuperação obtidas tanto para as amostras de sedimentos quanto para o material de referência utilizado (Quadro 3) foram satisfatórias (acima de $90 \%$ ) e estão de acordo com a literatura (Dhoun \& Evans, 1998; Weisz et al., 2000; Basílio et al., 2005; Pereira et al., 2007).

Embora pesquisas anteriores tenham evidenciado a presença de elevados teores de As total em sedimentos de diferentes regiões do Quadrilátero Ferrífero, estudos sobre a distribuição do As nas diferentes frações geoquímicas do sedimento permanecem escassos.

Pelos resultados encontrados neste estudo, podese verificar que os sedimentos da região apresentam quantidades significativas de As em frações consideradas biodisponíveis (ácido solúvel, redutível e oxidável), ainda que a maior parte do As encontrado esteja presente como fração residual, que não oferece risco ecotoxicológico em potencial a curto e médio prazos (Tessier et al., 1979; Förstner, 2002; Gleyzes et al., 2002; Filgueiras et al., 2004; Hlavay et al., 2004). A presença de elementos-traço em frações consideradas biodisponíveis constitui ainda uma evidência da contribuição antropogênica para a 
Quadro 3. Quantidades de As extraído pela aplicação do método BCR em 4 amostras de sedimentos e em material de referência SRM 2704 Buffalo River Sediment (NIST), utilizando a HG-AAS como método de detecção

\begin{tabular}{|c|c|c|c|c|c|c|c|}
\hline & \multicolumn{6}{|c|}{ As $\left(\mu g \mathbf{k g}^{-1}\right)^{(1)}$} & \multirow[b]{3}{*}{$\%$} \\
\hline & \multicolumn{4}{|c|}{ Etapa } & \multirow[b]{2}{*}{$\mathbf{P T}$} & \multirow[b]{2}{*}{ VC } & \\
\hline & 1 & 2 & 3 & 4 & & & \\
\hline $\mathrm{S} 1$ & $6,6 \pm 0,6$ & $56,0 \pm 2,3$ & $12,4 \pm 0,9$ & $344,1 \pm 18,3$ & $448,2 \pm 32,2$ & & 93 \\
\hline $\mathrm{S} 2$ & $209,9 \pm 11,7$ & $462,3 \pm 16,40$ & $181,4 \pm 14,6$ & $15.210 \pm 869,0$ & $17.090 \pm 1604$ & & 94 \\
\hline $\mathrm{S} 3$ & $161,6 \pm 13,9$ & $2.216 \pm 152,1$ & $483,6 \pm 43,85$ & $4.050 \pm 128,4$ & $7.055 \pm 558,2$ & & 98 \\
\hline $\mathrm{S} 4$ & $495,6 \pm 13,6$ & $4.494 \pm 113$ & $420,1 \pm 39,8$ & $13.093 \pm 1119$ & $18.669 \pm 627$ & & 99 \\
\hline MR & $<1,35$ & $4.311 \pm 227$ & $<1,86$ & $16.930 \pm 960$ & & 23.400 & 91 \\
\hline
\end{tabular}

(1) Média de 3 repetições \pm desvio-padrão; S1 a S4, amostras de sedimentos: S1 (Ribeirão Tripuí), S2 a S4 (Ribeirão do Carmo); MR, Material de referência. ${ }^{(2)}$ Valores precedido pelo símbolo < indicam limite de detecção; PT: teor pseudototal; VC: valor certificado; \%; percentagem de recuperação.

liberação destes elementos para o meio aquático, o que, no caso do Quadrilátero Ferrífero, se deve às atividades de mineração historicamente desenvolvidas na região.

\section{CONCLUSÕES}

1. Para possibilitar a utilização da HG-AAS como método de detecção de As nos extratos obtidos pela aplicação do método BCR de extração sequencial no fracionamento de As em sedimentos, diferentes condições de redução do arsênio( $V)$ foram necessárias, dependendo da etapa da extração. Nos extratos obtidos nas etapas 3 e 4, a adição de ácido ascórbico a $3 \%(\mathrm{~m} /$ v) e $2 \%(\mathrm{~m} / \mathrm{v})$, respectivamente, em associação ao iodeto de potássio a $1 \%(\mathrm{~m} / \mathrm{v})$ permitiu a redução e quantificação do As nas amostras, evitando interferências pelos agentes extratores oxidantes utilizados no método. A utilização do KI para a préredução do As nas etapas 1 e 2 permitiu simplificar a metodologia.

2. A utilização do método BCR para a extração de As a partir das amostras de sedimentos e do material de referência com valor certificado de As total, seguida pela aplicação das condições de redução do As(V) determinadas para cada uma das etapas e pela detecção do As por HG-AAS, forneceu boa porcentagem de recuperação. A metodologia constitui, portanto, uma ferramenta útil, simples, sensível e de baixo custo para o fracionamento e quantificação do As em sedimentos.

3. Embora neste trabalho as condições de préredução do $\mathrm{As}(\mathrm{V})$ tenham sido avaliadas especificamente nos extratos do método BCR, o estudo pode servir de base para que laboratórios de pesquisa ou rotina avaliem as condições de pré-redução do As(V) em diferentes extratos oxidativos, de acordo com as condições químicas e as necessidades de cada extrator utilizado, podendo lançar mão da HG-AAS para a quantificação do arsênio nestes extratos.

\section{AGRADECIMENTOS}

Os autores agradecem à FAPEMIG pelo apoio financeiro e ao CNPq pela bolsa concedida.

\section{LITERATURA CITADA}

BARRA, C.M.; SANTELLI, R.E.; ABRÃO, J.J. \& DE LA GUARDIA, M. Especiação de arsênio - uma revisão. Química Nova, 23:58-70, 2000.

BASILIO, M.S.; FRIESE, K.; LENA, J.C.; NALINI Jr, H. A. \& ROESER, H.M.P. Adsorção de $\mathrm{As}, \mathrm{Cu}, \mathrm{Pb}, \mathrm{e} \mathrm{Cr}$ na avaliação da capacidade de fixação de metais por resíduo de mineradoras de ferro. Química Nova, 28:822-828, 2005.

BORBA, R.P.; FIGUEIREDO, B.R. \& MATSCHULLAT, J. Geochemical distribution of arsenic in waters, sediments and weathered gold mineralized rocks from Iron Quadrangle, Brazil. Environ. Geol., 44:39-52, 2003.

BOUTAKHRIT, K.; CLAUS, R.; BOLLE, F.; DEGROODT, J.M. \& GOEYENS, L. Open digestion under reflux for determination of total arsenic in seafood by inductively coupled plasma atomic emission spectrometry with hydride generation. Talanta, 66:1042-1047, 2005.

BURGUERA, M. \& BURGUERA, J.L.J. Flow injectionelectrothermal atomic absorption spectrometry for arsenic speciation using Fleitmann reaction. J. Anal. At. Spectrom., 8:229-233, 1993

CORLEY, J. Best practices in establishing detection and quantification limits for pesticide residues in foods. In: LEE, P.W., ed. Handbook of residue analytical methods for agrochemicals. New York, John Wiley \& Sons, 2003. $1552 \mathrm{p}$. 
CREED, J.T.; MAGNUSON, M.L.; BROCKHOFF, C.A.; CHAMBERLAIN, I. \& SIVAGANESAN, M.J. Arsenic determination in saline waters utilizing a tubular membrane as a gas-liquid separator for hydride generation inductively coupled plasma mass spectrometry. J. Anal. At. Spectrom., 11:505-509, 1996.

DAVIDSON, C.M.; DUNCAN, A.L.; LITTLEJOHN, D.; URE, A.M. \& GARDEN, L.M. A critical evaluation of the threestage BCR sequential extraction procedure to asses the potential mobility and toxicity of heavy metals in industrially-contaminated land. Anal. Chim. Acta, 363:45$55,1998$.

DEDINA, J. \& TSALEV, D.L. Hydride generation atomic absorption sspectrometry. Chichester, John Wiley \& Sons, 1995. 526p.

DESCHAMPS, E.; CIMINELI, V.S.T.; LANGE, F.T.; MATSCHULLAT, J.; RAUE, B. \& SCHIMIDT, H. Soil and sediment geochemistry of the Iron Quadrangle, Brazil: The case of arsenic. J. Soil Sediments, 2:216-222, 2002.

DHOUM, R.T. \& EVANS, G.J. Evaluation of uranium and arsenic retention by soil from a low level radioactive waste management site using sequential extraction. Appl. Geochem., 13:415-420, 1998.

FENG, Y.L.; CHEN, H.Y.; TIAN, L.C. \& NARASAKI, H. Offline separation and determination of inorganic arsenic species in natural water by high resolution inductively coupled plasma mass spectrometry with hydride generation combined with reaction of arsenic (V) and Lcysteine. Anal. Chim. Acta, 375:167-175, 1998.

FILGUEIRAS, A.V.; LAVILLA, I. \& BENDICHO, C. Chemical sequential extraction form metal partitioning in environmental solid samples. J. Environ. Monitoring, 4:823-857, 2004

FÖRSTNER, U. Traceability of sediment analysis. Trends Anal. Chem., 23:217-236, 2004

GISMERA, M.J.; LACAL, J.; SILVA, P.; GARCIA, R.; SEVILLA, M.T. \& PROCOPIO, J.R. Study of metal fractionation in river sediments. A comparison between kinetic and sequential extraction procedures . Environ. Poll., 127:175182,2004

GLEYZES, C.; TELLIER, S. \& ASTRUC, M. Fractionation studies of trace elements in contaminated soils and sediments: A review of sequential extraction procedures. Trends Anal. Chem., 21:451-467, 2002.

GONÇALVES, J.A.C.; LENA, J.C.; PAIVA, J.F.; NALINI Jr., H.A. \& PEREIRA, J.C. Arsenic in the groundwater of Ouro Preto (Brazil): Its temporal behavior as influenced by hydric regime and hydrology. Environm. Geol., 53:785$793,2007$.

HLAVAY, J.; PROHASKA, T.; WEISZ, M.; WENZEL, W.W. \& STINGEDER, G.J. Determination of trace elements bound to soil and sediment fractions (IUPAC Technical Report). Pure Appl. Chem., 76:415-442, 2004.

KUMAR, A.R. \& RIYAZUDDIN, P. Mechanism of volatile hydride formation and their atomization in hydride generation atomic absorption spectrometry. Anal. Sci., 21:1401-1408, 2005
KUMARESAN, M. \& RIYAZUDDIN, P. Overview of speciation chemistry of arsenic. Curr. Sci., 80:837-845, 2001.

LONG, G.L. \& WINEFORDNER, J.D. Limit of detection. A closer look at the IUPAC definition. Anal. Chem., 55:712$724,1983$.

MARGUÍ, E.; SALVADÓ, V.; QUERALT, I. \& HIDALGO, M. Comparison of three-stage sequential extraction and toxicity characteristic leaching test to evaluate metal mobility in mining wastes. Anal. Chim. Acta, 524:151159, 2004

MATSCHULLAT, J.; BORBA, R.P.; DESCHAMPS, E.; FIGUEIREDO, B.R.; GABRIO, T. \& SCHWENK, M. Human and environmental contamination in the Iron Quadrangle, Brazil. Appl. Geochem., 15:193-202, 2000.

MELLO, J.W.V.; ROY, W.R.; TALBOTT, J.L. \& STUCKI, J.W. Mineralogy and arsenic mobility in arsenic-rich brazilian soils and sediments. J. Soil Sediments, 6:9-19, 2006.

NIELSEN, S. \& HANSEN, E.H. Determination of As(III) and $\mathrm{As}(\mathrm{V})$ by flow injection-hydride generation-atomic absorption spectrometry via on-line reduction of $\mathrm{As}(\mathrm{V})$ by KI. Anal. Chim. Acta, 343:5-17, 1997.

PEREIRA, J.C.; SILVA, A.K.G.; NALINI Jr., H.A.; SILVA, E.P. \& LENA, J.C. Distribuição, fracionamento e mobilidade de elementos-traço em sedimentos superficiais. Química Nova, 30:1249-1255, 2007.

PERTSEMLI, E. \& VOUTUSA, D. Distribution of heavy metals in Lakes Doirani and Kerkini, Northern Greece. J. Haz. Mat., 148:529-537, 2007.

PUEYO, M.; SASTRE, J.; HERNANDEZ, E.; VIDAL, M.; LOPEZ-SANCHEZ, J.F. \& RAURET, G. Prediction of trace element mobility in contaminated soils by sequential extraction. Environ. Qual., 32:2054-2066, 2003.

RAURET, G.; LOPEZ-SANCHEZ, J.F.; SAHUQUILLO, A.; RUBIO, R.; DAVIDSON, C.; URE, A. \& QUEVAUVILLER, $\mathrm{Ph}$. Improvement of the BCR three step sequential extraction procedure prior to the certification of new sediment and soil reference materials. J. Environ. Monitoring, 1:57-61, 1999.

RISNES, A. \& LUND, W. Comparison of systems for eliminating interferences in the determination of arsenic and antimony by hydride generation inductively coupled plasma atomic emission spectrometry. J. Anal. At. Spectrom., 11: 943-948, 1996.

SAHUQUILLO, A.; LÓPEZ-SANCHEZ, J.F.; RUBIO, R.; RAURET, G.; THOMAS, R.P.; DAVIDSON C.M. \& URE, A.M. Use of a certified reference material for extractable trace metals to assess sources of uncertainty in the BCR three-stage sequential extraction procedure. Anal. Chim. Acta, 382:317-237, 1999 .

SHRAIM, A.; CHISWELL, B. \& OLSZOWY, H. Speciation of arsenic by hydride generation - atomic absorption spectrometry (HG-AAS) in hydrochloric reaction medium. Talanta, 50:1109-1127, 1999.

TAKASE, I.; PEREIRA, H.B.; LUNA, A.S.; GRINBERG, P. \& CAMPOS, R.C. A geração química de vapor em espectrometria atômica. Quimica Nova, 25:1132-1144, 2002. 
TESSIER, A.; CAMPBELL, P.G.C. \& BISSON, M. Sequential extraction procedure for the speciation of particulate trace metals. Anal. Chem., 51:844-851, 1979.

TOKALIOGLU. S.; KARTAL, S. \& ELÇI, L. Determination of heavy metal and their speciation in lake sediments by flame atomic absorption spectrometry after a four-stage sequential extraction procedure. Anal. Chim. Acta, 413:3340, 2000 .

URE, A.M.; QUEVAUVILLER, Ph.; MUNTAU, H. \& GRIEPINK, B. Speciation of heavy metals in soils and sediments. An account of the improvement and harmonization of extraction techniques undertaken under the auspices of the BCR of the Commission of the European Communities. Inter. J. Environ. Anal. Chem., 51:133-151, 1993.
WARD, M. \& GRAY, A. Vapor Generation Accessory VGA-77. Operational manual. Varian Australia Pty, Nov., 1994. 55p. (Publication, 85101047 00)

WEISZ, M.; POLYAK, K. \& HLAVAY, J. Fractionation of elements in sediment samples collected in rivers and harbors at Lake Balaton and its catchment area. Microchem. J., 67:207-217, 2000.

ZEMBERYOVÁ, M.; BARTEKOVÁ, J. \& HAGAROVÁ, I. The utilization of modified BCR tree-step sequential extraction procedure for the fractionation of $\mathrm{Cd}, \mathrm{Cr}, \mathrm{Cu}, \mathrm{Ni}, \mathrm{Pb}$ and $\mathrm{Zn}$ in soil reference materials of different origins. Talanta, 70:973-978, 2006. 
